\title{
STATUEN IN VERBANNUNG ÄGYPTISCHER STATUENEXPORT IN DEN VORDEREN ORIENT UNTER AMENOPHIS III. UND IV.
}

Von Irene Forstner-Müller, Wolfgang Müller und Karen Radner

\section{§1. EinLeitung}

Der Gegenstand der vorliegenden Untersuchung ist zunächst ein klar überliefertes, vordergründig relativ einfaches Phänomen, nämlich der Austausch von Statuen zwischen den Großmächten des Vorderen Orients und Ägypten während der 18. Dynastie, wobei dieser Austausch sehr einseitig vonstatten ging, nämlich von Ägypten in die Länder des Vorderen Orients.

Die quantitative Komponente wurde mit ersten Interpretationsansätzen erst kürzlich umfangreich vorgelegt. ${ }^{1}$ Basierend auf dieser Bestandsaufnahme der schriftlichen Quellen soll im folgenden erläutert werden, weshalb dieses Phänomen mehr darstellt als einen wie auch immer gearteten „Kunsthandel“, wie er für das Mittlere Reich angenommen worden ist. ${ }^{2}$ Statuen werden zum Gegenstand des diplomatischen Verkehrs ${ }^{3}$ und sind Teile des nicht zwingend notwendigen Anteils an Gütern, der Luxusartikel und „cultural items“. 4

Wesentlich sind dabei die kulturellen Perspektiven der beiden Austauschpartner. Aus ägyptischer Sicht handelt es sich um eine Dislozierung von Sta- tuen. $\mathrm{Zu}$ hinterfragen ist, aus welchem Kontext sozialer, religiöser und künstlerischer Natur die Kunstwerke in einen neuen Kontext, dessen Beschaffenheit im Gegenzug ebenfalls erforscht werden muss, verbracht wurden. Gerade angesichts des großen Anteils an kleinen, also tragbaren Statuen muss besonderes Augenmerk auf die Tatsache dieses Verbringens selbst gerichtet werden, da ägyptische Statuen üblicherweise nicht statisch verstanden wurden, sondern vielfach erst in ihrer dynamischen Komponente, bei Prozessionen und dergleichen, für die Kultteilnehmer in ihrer Potenz erfahrbar wurden. Durch die Bewegung wurden erst die narrativen Zusammenhänge zwischen den einzelnen Kunstwerken hergestellt, ${ }^{5}$ die einzelnen Stationen der „liturgischen Topographie“ ${ }^{66} \mathrm{im}$ mythologischen Sinne verwoben und für die Teilnehmer realisiert.

\section{§2. Ägyptische Statuen als Gabe AN die ORIEN- talischen Herrscher zUR Zeit der 18. Dynastie}

In der sogenannten Amarnakorrespondenz ${ }^{7}$ der ägyptischen Pharaonen der 18. Dynastie mit den asiatischen Großmächten Babylonien, Mitanni und

\footnotetext{
1 Z. Cochavi-Rainey/C. Lilyouist, Royal Gifts in the Late Bronze Age Fourteenth to Thirteenth Centuries B.C.E. Selected Texts Recording Gifts to Royal Personages. Beer Sheva XIII, Beer Sheva 1999. Vgl. D. Panagiotopoulos, Tributabgaben und Huldigungsgeschenke aus der Levante. Die ägyptische Nordexpansion in der 18. Dynastie aus strukturgeschichtlicher Sicht, $\ddot{A} \mathcal{E}^{2} L \quad 10$ (2000), 139-158 zu einer ökonomisch machtpolitischen Interpretation der Amarnakorrespondenz.

2 W. Helck, Ägyptische Statuen im Ausland. Ein chronologisches Problem, UF 8 (1976), 114.

3 C. Zaccagnini, The Interdependence of the Great Powers, in: R. Cohen/R. Westbrook (Hg.), Amarna Diplomacy. The Beginnings of International Relations, Baltimore/ London 2000, 147-149. Grundlegend zu den diplomatischen Beziehungen der Großmächte der Spätbronzezeit ist M. Liverani, Prestige and Interest. International Relations in the Near East ca. 1600-1100 B.C. History of the Ancient Near East. Studies 1, Padua 1990.

${ }^{4}$ R. Cohen/R. Westbrook, Conclusion: The Beginnings of International Relations, in: Cohen/Westbrook, $o p$. cit., 226.
}

\footnotetext{
5 A. Egberts, Praxis und System, in: 3. Ägyptologische Tempeltagung. Hamburg 1.-5. Juni 1994. НÄB 33 (1995), 15-17 zum Fest von Beheded. Auch vorderasiatische Statuen werden durch ihre Rolle in Kultprozessionen definiert, s. dazu B. Pongratz-LeIsten, Ina Šulmi Irub. Die kulttopographische und ideologische Programmatik der akìtu-Prozession in Babylonien und Assyrien im I. Jahrtausend v. Chr. Baghdader Forschungen 16, Mainz 1994 und W. Sallaberger, Das Erscheinen Marduks als Vorzeichen: Kultstatue und Neujahrsfest in der Omenserie Šumma ālu, ZA 90 (2000), 227-262.

6 Egberts, op. cit., 30.

71887 wurde in Tell el-Amarna, dem alten Achet-Aton, ein Tontafelarchiv gefunden, bei dem es sich um Teile der in akkadischer Sprache abgefaßten diplomatischen Korrespondenz der Pharaonen der 18. Dynastie handelte. Die ältesten der rund 350 Texte stammen aus dem 13. Regierungsjahr Amenophis' III., der Großteil aus der Regierungszeit Amenophis' IV. (Echnaton) und die letzten Texte aus dem ersten Regierungsjahr Tutanchamuns. Die Tafeln wurden in einem nach Ausweis der Inschriften „Ort der Briefe des Pharaos“ benannten
} 
Hatti finden sich wiederholt Hinweise auf die Expedierung von ägyptischen Statuen in diese Länder. Umgekehrt ist der Versand von Statuen aus den Ländern des vorderen Orients nach Ägypten jedoch nicht zu belegen, sieht man von einer Ausnahme ab. Die Statue der Göttin Šawuška (Ištar) von Nineveh wird je einmal von den Mitannikönigen Šuttarna II. und Tušratta, seinem Sohn, zur Heilung von Amenophis III. nach Ägypten geschickt. ${ }^{8}$ Anders aber als die temporär zu Heilzwecken ins Ausland verbrachte Statue der Šawuška sollten die ägyptischen Statuen aber auf Dauer in der Fremde bleiben.

Die ausführlichste Quelle für Statuen ägyptischer Herkunft, die in den Vorderen Orient geschickt werden, ist der Brief EA $14,{ }^{9}$ die in Amarna aufgefundene Kopie eines Schreibens des Echnaton an den babylonischen König Burna-Buriaš
II., das die anläßlich seiner Verheiratung mit einer Tochter des Burna-Buriaš nach Babylon gesandten Geschenke im Detail auflistet. Die reichhaltigen Gaben umfaßten kostbarste Möbel, reichen Schmuck, unzählige Toilettgegenstände, prunkvolle Textilien, vor allem aus Leinen, Gefäße jeder Art mit edlen Aromata und Salbölen als Inhalt, Streitwägen, sowie verschiedene Statuen und Schiffsmodelle, deren Beschreibungen uns im vorliegenden Kontext besonders interessieren. Nach „einer großen, mit Gold überzogenen Statue des Königs und ihren mit Silber überzogenen Fußschemel ${ }^{\text {"10 }}$ sind „eine mit Gold überzogene weibliche Statue der Gattin des Königs" ${ }^{\text {"11 }}$ und dann „eine mit Gold überzogene weibliche Statue der Tochter des Königs “"12 genannt. An antropomorphen Statuen ist als Teil der Brautgabe weiters ,ein steinerner Krummbeiniger, in seiner Hand ein Gefäß $3^{\text {“13 }}$ auf-
Komplex in Echnatons neuer Hauptstadt Achet-Aton, dem heutigen Amarna, gefunden, wo sie bei der Verlagerung der Hauptstadt nach Memphis zum Regierungsantritts Tutanchamuns aus uns nicht bekannten Gründen zurückgelassen, vielleicht auch nur vergessen wurden. Aus den vorhandenen Texten geht hervor, daß bereits Tuthmosis IV. mit den Herrschern Babyloniens und des Mitannireiches Briefe ausgetauscht hatte. Diese Korrespondenz, wie auch der Großteil der Briefe aus der Regierungszeit Amenophis' III., ist uns aber nicht bekannt; es ist anzunehmen, daß diese Briefe, aus welchen Gründen auch immer, von Echnaton bei seinem Umzug von Theben nach Amarna nicht überführt wurden oder aber zur Gänze von Tutanchamun nach Memphis verbracht wurden. Neben der Korrespondenz mit Ägyptens Vasallen in der Levante sind uns die an den ägyptischen Pharao gerichteten Briefe der Herrscher der Großmächte dieser Zeit erhalten. Es sind dies Babylonien, das Hethiterreich und das Reich der hurritischen Mitanni, Assyrien sowie Zypern (Alašia) und das Land Arzawa an der anatolischen Südküste. Darüber hinaus finden sich unter den Briefen auch einige Kopien von Briefen des Pharao. Die Texte sind allesamt in Keilschrift auf Tontafeln niedergeschrieben, wobei die babylonische Sprache verwendet wurde, mit Ausnahme zwei Briefe in hethitischer und ein hurritisches Schreiben. Die Amarna-Korrespondenz wurde zuletzt bearbeitet von W. L. MORAn, The Amarna Letters, Baltimore/London 1992. EA bezeichnet im folgenden die Signatur der Amarna-Briefe nach J. A. KnUdTzOn, Die El-AmarnaTafeln. Vorderasiatische Bibliothek 2, Leipzig 1907-15, wie sie ebenfalls von Moran verwendet wird. Neben der internationalen Korrespondenz wurden in Amarna auch literarische und lexikographische Texte gefunden; erwähnenswert sind dabei besonders einige babylonische Mythen und ein hurritisches Märchen. Diese Texte sowie einige Tafeln mit Schreibübungen legen nahe, daß es in Achet-Aton eine Schule zur Ausbildung von Keilschriftschreibern gab. Diese Texte wurden jüngst von SH. IzRE'EL, The Amarna Scholarly Tablets. Cuneiform Monographs 9, Groningen 1997, vorgelegt.

8 EA 23. Dazu I. WEgner, Gestalt und Kult der IštarŠawuška in Kleinasien. AOAT 36, Kevelaer/NeukirchenVluyn 1981, 65, G. Wilhelm, Grundzüge der Geschichte und Kultur der Hurriter. Grundzüge 45, Darmstadt 1982, 40-41; L. KákosY, Heilsstatuen in den Tempeln, in: 3. Ägyptologische Tempeltagung Hamburg 1.-5. Juni 1994, HÄB 33 (1995), 93 und ZACCAGNINI, op. cit. (s. o. Anm. 3), 146.

9 Zuletzt bearbeitet von Cochavi-Rainey/LilyQuist, op. cit. (s. o. Anm. 1), 7-38 und 219-228.

10 EA 14 ii 11-12: 1 ALAM GAL ša KÙ.GI GAR.RA ša LUGAL (12) ù šu-pá-al GİR.MEŠ-šu KÙ.BABBAR GAR.RA. Cochavi-Rainey/LilyQuist, op. cit. (s. o. Anm. 1), 212 vergleichen diese Beschreibung mit einer vergoldeten, auf einem rechteckigen Sockel angebrachten Holzstatue des Tutanchamun aus seinem Grabinventar, s. ibid., 48-49 mit Abb. 11-12.

11 EA 14 ii 13: 1 dLÁMA KÙ.GI GAR.RA ša DAM LUGAL. Für die Verwendung von ALAM und 'LÁMA als Gegensatzpaar „männliche Statue“ - „weibliche Statue“ s. Moran, op. cit. (s. Anm. 7), 35. Vgl. auch „21 steinerne weibliche Statuen“" in EA 14 iii 59: 21 ¿LÁMA ša $\mathrm{NA}_{4}$.

12 EA 14 ii 14: 1 dLÁMA KÙ.GI GAR.RA ša DUMU.MÍ-ti LUGAL.

13 EA 14 iii 60: 1 LÚ.pé-eš-šú-ú ša NA 4 ki-ir-re-tù ina [Š]Uti-šu. Cochavi-Rainey/LilyQuist, op. cit. (s. o. Anm. 1), 212 vergleichen diese Beschreibung überzeugend mit der Steinstatue eines Mannes mit verkrüppelten Beinen, der auf seiner linken Schulter ein Gefäß aufstützt; Abb. s. ibid., Taf. 2. 
gelistet, eventuell eine Darstellung des Gottes Bes. ${ }^{14}$ Die Figur einer „silbernen Äffin mit ihrer Tochter auf ihrem Scho $\beta^{\text {“15 }}$ ist in einer Sequenz von Gefäßen aufgelistet; Sally Dunhams Identifizierung dieser Statuenbeschreibung mit einem Gefäßtyp, wie er durch eine Alabastervase des Pharaos Meryen-rec der 6. Dynastie repräsentiert wird, erscheint deshalb umso überzeugender. Die genannte Vase setzt sich aus einer Affenmutter mit ihrem Jungen zusammen; die Äffin hält das Junge, das auf ihrem Schoß sitzt, in beiden Armen, während das Jungtier die Mutter mit allen Vieren umschlingt. ${ }^{16}$ Auch die „steinernen Onager und ein identisches Junges ${ }^{\text {“17 }}$ werden inmitten von Gefäßen aufgelistet, sodaß man auch hier an eine tiergestaltige Vase denken sollte. Besonders interessant sind aber „ein mit Gold überzogenes Schiff aus Zedernholz, zusammen mit allen Gerätschaften, und sechs kleine Schiffe, die man zieht" ${ }^{18}$

Bemerkenswert ist, daß Statuen und Barken, die wohl ursprünglich zum Tempelinventar gehört haben müssen, überhaupt außer Landes gebracht wurden. Dies ist umso auffälliger, wenn man einen Blick auf die reichen Geschenke wirft, die Ramses II., seine Gattin, seine Mutter und sein Sohn nach dem Abschluß des Friedensvertrags zwischen Ägyptern

14 Für den Vorschlag, den Krummbeinigen mit dem Gott Bes zu identifizieren, s. CAD K 409 sub kirru A 1.a., zustimmend Moran, op. cit. (s. Anm. 7), 37.

${ }^{15}$ EA 14 ii 48: 1 pa-gu $u_{5}$-tù ù DUMU.MÍ-šu i-na sú-n[i]-ši ša KÙ.BABBAR.

${ }^{16} \mathrm{~S}$. Dunham, The Monkey in the Middle, ZA 75 (1985), 260 mit Abb. 9, s. auch W. C. HAYEs, The Scepter of Egypt: A Background for the Study of Egyptian Antiquities in the Metropolitan Museum of Art. Part I: From the Earliest Times to the End of the Middle Kingdom, New York 1953, 128 Abb. 78, H. G. FISCHER, Another Pitheomorphic Vessel of the Sixth Dynasty, JARCE XXX (1993), 1-7. Die Affenmutter mit Jungem ist ein rein ägyptisches Motiv; Darstellungen weiblicher Affen kommen in der Kunst des Vorderen Orients kaum vor, während der männliche Affe besonders in der altbabylonischen Zeit häufig als Symbol maskuliner Kraft im Zusammenhang mit der Göttin Ištar dargestellt wird, s. A. Намото, Der Affe in der altorientalischen Kunst. Forschungen zur Anthropologie und Religionsgeschichte 28, Münster 1995, 74. In der mittelbabylonischen Zeit sind Affendarstellung im Vorderen Orient allgemein selten zu belegen, s. ibid., 52. Dazu siehe unten, § 4.

17 EA 14 iii 49: [x] NA 4 pa-re-e ša NA 4 [ù] 1 șé-[eh-rù] ki šašu-ma.

18 EA 14 ii 17-18: 1 GIŠ.MÁ ša GIŠ.EREN e-re-ni KÙ.GI und Hethitern an den hethitischen Königshof sandten. In den begleitenden Briefen ${ }^{19}$ werden zwar diverse Gefäße aus Edelmetall, prunkvolle Textilien und Juwelen aufgezählt, ${ }^{20}$ Statuen und Barken fehlen aber völlig.

Auffällig ist, daß die von Amenophis III. offenbar sehr forciert betriebene Praxis des Statuenexports unter seinem Nachfolger Echnaton stark eingeschränkt wurde. Dies können wir daraus erschließen, daß sowohl der Mitanni- wie auch der Hethiterkönig sich in ihren Briefen darüber beschwerten, daß die von Amenophis III. versprochene Statuenlieferungen nach der Thronbesteigung durch Echnaton nun nicht eintrafen. So hatte Amenophis III. Tušratta von Mitanni die Übersendung zweier massiv goldener Statuen versprochen, von denen eine den König selbst, die andere seine nach Ägypten verheiratete Tochte Dadu-Hepa darstellen sollte. Amenophis selbst sei es gewesen, so Tušratta in seinen Briefen, der bloße Goldstatuen als zu minder befunden hätte und deshalb die Lieferung von goldenen Statuen mit Lapislazulidekor in Aussicht gestellt hätte. Sein Nachfolger Echnaton enthielt nun dem Tušratta diese Statuen vor und meinte, daß durch die Übersendung eines Paares vergoldeter Holzstatuen das Versprechen abgegolten uh-hu-zu qa-du gáb ú-nu-te.MEŠ (18) ù 6 GIŠ.MÁ.HI.A ruku-bu sé-hi-ru-ti ša i-ša-ad-dá!-du. Gerade die prächtige Ausstattung könnte eine Deutung dieser Schiffe als Götterbarken, wie sie bei Prozessionen gebräuchlich waren, nahelegen.

19 Die Briefe, die in der hethitischen Hauptstadt Hattuša gefunden wurden, sind das Gegenstück zur Amarnakorrespondenz der 18. Dynastie. Sie wurden während der Regierungszeit Ramses' II. aus Ägypten an den Hof des Hethiterkönigs Hattušili III., des Onkels und zweiten Nachfolgers seines Gegenspielers Muwatalli bei der Schlacht von Qadeš, gesandt. Mit Ausnahme einiger Briefentwürfe oder -kopien ist uns der hethitische Teil dieser Korrespondenz nicht erhalten; dieser wäre in Ramses' Hauptstadt Piramesse zu vermuten, wurde aber bisher bei den dortigen Grabungen nicht zutage gefördert. Die Briefe aus Hattuša sind überwiegend in babylonischer Sprache abgefaßt, nur acht Stück bedienen sich des Hethitischen. Sie wurden zuletzt bearbeitet von E. EDEL, Die ägyptisch-hethitische Korrespondenz aus Boghazköi in babylonischer und hethitischer Sprache. Abhandlungen der Rheinisch-Westfälischen Akademie der Wissenschaften 77, Opladen 1994.

${ }^{20}$ Die Beschreibungen der Geschenke von Ramses II. und seiner Angehörigen an die hethitische Königsfamilie sind bei Cochavi-RAiney/Lilyquist, op. cit. (s. o. Anm. 1), 195-203 gesammelt. 
wäre, sehr zum Mißfallen Tušrattas, der in mehreren Briefen seine Forderung nach der Lieferung der Statuen wiederholte. Einer dieser Briefe, EA 27, ist an Echnaton gerichtet, ein weiterer, EA 26, an Amenophis' Witwe Teye, die Echnaton die Rechtmäßigkeit von Tušrattas Forderung bestätigen sollte. ${ }^{21}$ Auch Šuppiluliuma von Hatti forderte mit einem Brief, EA 41, einen ägyptischen Pharao $^{22}$ auf, sich an die mit seinem Vater getroffene Abmachung zu halten und endlich zwei goldene Statuen, wobei die eine sitzend, die andere stehend sein solle, sowie zwei silberne Frauenstatuen zu schicken. ${ }^{23}$ Wenn man die Vernachlässigung der Wünsche des Tušratta von Mitanni durchaus mit der fortschreitenden Schwächung dieses vormals dominanten Reiches ${ }^{24}$ in Verbindung bringen kann, so erschiene es doch höchst undiplomatisch, auch die zunehmend mächtiger werdenden Hethiter ${ }^{25}$ durch die Vorenthaltung bereits versprochener Statuen zu verstimmen. Man darf annehmen, daß hier weniger böse Absicht als vielmehr ein Engpaß an verfügbaren Statuen zum Tragen kommt.
21 EA 27: 19-34: ù ALAM.MEŠ ša KÙ.GI ša-ap-gu-tu ${ }_{4}$ up-

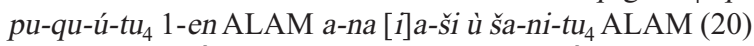
a-na ALAM MÍ.da-a-duHhé-e-pa DUMU.MÍ-ia a-šar a-bi-

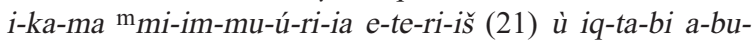
ka-ma muš-šèr a-na ša KÙ.GI-ma ša-pí-ik-ta up-pu-uq-ta na-ta-a-an-sú-nu-me (22) ù ša $\mathrm{NA}_{4}$.ZA.GİN.KUR a-na-andin-ak-kum-me ù KÙ.GI ap-pu-na ša-na-a ma-a'-ta ú-nu-ta (23) [ša] pa-ta la i-šu-ú it-ti ALAM.MEŠ a-na-an-din-akkum-me ù KÙ.GI ša ALAM.MEŠ LÚ.DUMU.MEŠ KIN-ia (24) [g]áb-bi-i-su-nu-ma ša i-na KUR.mi-iṣ-ri-i aš-bu i-na IGI.MEŠ-šu-nu i-ta-am-ru ù ALAM.MEŠ a-bu-ka-ma (25) [a]-na pa-ni LÚ.DUMU.MEŠ KIN-ia a-na ši-ip-ki ut-te-eer-šu-nu i-te-pu-us-sú-nu ig-ta-mar-šu-nu (26) [u]s-sé-ekki-šl-nu ù ki-i a-na ši-ip-ki du-ur-ru LÚ.DUMU.MEŠ KIN-ia [i-n]a IGI.MEŠ-šu-nu i-tam-ru (27) ù ki-i kám-ruma za-gu-ú i-na IGI.MEŠ-šu-nu i-ta-am-ru (28) ù KÙ.GI ša-nu-ú ma-a'-du ša pa-ta la i-šu-ú ša a-na ia-ši ú-še-e-ebbi-lu uk-te-li-im-ma (29) ù iq-ta-bi a-na LÚ.DUMU.MEŠ KIN-ia a-nu-um-ma ALAM.MEŠ ù a-nu-um-ma KÙ.G[I] ma-a'-ta ù ú-nu-ta (30) ša-a pa-ta la i-šu-ú ša a-na ŠEŠ-ia ú-še-bi-lu ù i-na IGI.MEŠ-gu-nu am-ra-e-me (31) ù LÚ.DUMU.MEŠ KIN-ia i-na IGI.MEŠ-šu-nu i-ta-am-ru (32) ù i-na-an-na ŠEŠ-ia ALAM.MEŠ up-pu-qu-ú-du ša abu-ka ú-še-e-eb-bé-lu la du-še-bi-la (33) ù ša GIŠ.MEŠ uhhu-zu-du du-ul-te-e-bi-la ú-nu-ta ša a-bu-ka a-na ia-ši ú-šee-eb-bé-lu (34) [I]a du-še-e-bi-lam-ma ù du-ul-te-me-eș dan-níš-ma (für die Umschrift s. P. ADLER, Das Akkadische des Königs Tušratta von Mitanni. AOAT 201, Kevelaer/Neukirchen-Vluyn 1976, 214): „Massiv gegossene Statuen aus Gold, eine Statue von mir [dazu Moran, op. cit. (s. o. Anm. 7), 89 Anm. 6] und eine zweite Statue als Statue meiner Tochter Dadu-Hepa, habe ich von Deinem Vater Mimmurija erbeten. Und Dein Vater hat gesagt: ,Sprich nicht davon, ihnen nur massiv gegossene Statuen aus Gold zu geben. Ich werde Dir Statuen aus (Gold und) Lapislazuli geben, und darüberhinaus noch mehr Gold und Gerätschaften sonder Zahl werde ich Dir mit den Statuen geben." Und das Gold für die Statuen haben alle meine Boten, die sich in Ägypten aufhielten, mit eigenen Augen gesehen, und die Statuen hat Dein Vater in Gegenwart meiner Boten dem Guß zugeführt und sie vollständig aus reinem Gold gemacht. Meine Boten haben mit eigenen Augen gesehen, daß sie dem Guß zugeführt wurden, und mit eigenen Augen haben sie gesehen, daß sie vollständig aus reinem Gold waren. Dann zeigte er viel zusätzliches Gold sonder Zahl, das er mir übersenden wollte, und sagte $\mathrm{zu}$ meinen Boten: ,Hier also sind die Statuen, hier also sind das viele Gold und die Gerätschaften sonder Zahl, das ich meinem Bruder übersenden will, seht mit euren eigenen Augen!' Und meine Boten haben es mit eigenen Augen gesehen. Doch jetzt hast Du, mein Bruder, die massiven Statuen, die Dein Vater übersenden wollte, nicht übersandt, sondern hast (Statuen) aus Holz, die (nur mit Gold) überzogen sind, übersandt. Die Gerätschaften, die Dein Vater mir übersenden wollte, hast Du (auch) nicht übersandt! So hast Du (alles) sehr verringert!“ Ähnlich, aber kürzer in EA 26: 35-41, s. ADLER, op. cit., 208 für die Umschrift.

${ }^{22}$ Der sicher fehlerhafte Name Hurija kann den „Thronnamen aller Amarna-Pharaonen (Amenophis III. ausgenommen)“ wiedergeben, s. C. KüHne, Die Chronologie der internationalen Korrespondenz von El-Amarna. AOAT 17 (1973), 101, also entweder Echnaton, Tutanchamun oder Semenchkare. Zwar argumentierten G. Wilhelm/J. Boese, Absolute Chronologie und die hethitische Geschichte des 15. und 14. Jahrhunderts v. Chr., in: P. Åström (Hg.), High, Middle or Low 1, Gothenburg 1987, 97 aus philologischen und „fehlerpsychologischen“ Überlegungen für letzteren, doch scheut sich die Forschung insgesamt, sich auf einen bestimmten Pharao festzulegen, s. zuletzt H. KLENGEL, Geschichte des hethitischen Reiches. HdO I 34, Leiden/New York/Köln 1999, 139 sub A 10.

23 EA 41: 25-27: [2 șa]-al-ma-a-ni ša KÙ.GI 1-en (26) [lizziz] 1-en li-ši-ib ù 2 ALAM.MEŠ ša MÍ.MEŠ (27) [ša KÜ.BABB]AR-ma „[Zwei St]atuen aus Gold - eine [möge stehen], eine möge sitzen - und zwei Statuen von Frauen aus Silber.“

24 Zum Niedergang Mitannis unter Tušratta s. C. KüHNE, Imperial Mittani: An Attempt at Historical Reconstruction, SCCNH 10 (1999), 218-220.

${ }_{25} \mathrm{Zu}$ Šuppiluliuma I., dem ersten Herrscher der sog. hethitischen „Großreichszeit“, s. zuletzt KLENGEL, op. cit. (s. o. Anm. 22), 135-167. 
Die Erfahrungen des babylonischen Königs Burna-Buriaš II. mit Echnaton sind in dieser Beziehung nach unserem Kenntnisstand weniger ärgerlich als die der Könige von Mitanni und Hatti, vielleicht auch deshalb, weil er sich weniger wählerisch zeigte. So ersuchte er Echnaton in einem Brief, EA 10, darum, seine Handwerker das Abbild eines wilden Wasser- oder Landtiers herstellen zu lassen, das so lebensecht sein sollte, daß sich seine Haut nicht von einem lebenden Tier zu unterscheiden sei. Da die Sache dringlich ist, betonte er, daß man ihm auch alte Exemplare solcher Statuen schicken könnte, sofern welche vorhanden wären, und die neugefertigten später schicken könnte. ${ }^{26}$

Nach dem Ausweis der Amarnakorrespondenz wurden die in den Vorderen Orient verschickten ägyptischen Statuen ganz offensichtlich ihrer Zierfunktion wegen geschätzt; sie dienten der Ausstattung der Paläste der vorderasiatischen Könige. Es gibt keinerlei Anhaltspunkte dafür, daß die Statuen

26 EA 10: 32-42: NAGAR.MEŠ le-ú-tu i-tu-ka i-ba-aš-šu-ú (33) ú-ma-ma lu ša ta-ba-li lu ša ÌD (34) a-na pi-i ba-al-ṭi lima-aš-ši-lu-ma ma-aš-ku (35) ki-i ša ba-al-ti-ma lu e-pu-uš DUMU ši-ip-ri-ka li-il-qa-a (36) ù šum-ma la-bi-ru-tu ip-šu-

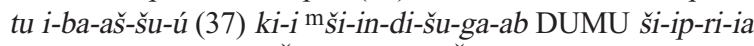
ik-ta-al-da-ku (38) GIŠ.GIGIR.M[EŠ] ki-i ka-al-le-e ha-muut-ta li-iš-ša-am-ma (39) a-na mu-uh-hi-ia li-ik-šu-da (40) ù e[š-š $] u-t i$ ar-ku-ti li-pu-šu-ma (41) ki-i DUMU ši-ip-ri-ia ù DUMU ši-ip-ri-ka il-la-ka (42) it-ti a-ha-mi-iš li-il-qú-ni „Es gibt fähige Zimmerleute, wo Du bist. Ein wildes Tier sowohl des trockenen Landes als auch des Flusses sollen sie lebensecht darstellen, und die Haut soll gleich einem lebendigen Tier gefertigt sein! Dein Bote soll (es) bringen! Wenn aber fertige alte vorhanden sind, dann lasse, sowie mein Bote Šindišugab Dich erreicht, ihn wie per Eilpost sofort Wagen borgen und mich erreichen. Und neue (Tiere) sollen sie für die Zukunft herstellen, und wenn mein Bote und Dein Bote (zu mir) kommen, dann sollen sie (sie) gemeinsamen herholen." Vgl. die Statuen von Wasser- und Landtieren an den Toren assyrischer Paläste, s. dazu S. M. Maul, Der Sieg über die Mächte des Bösen. Götterkampf, Triumphrituale und Torarchitektur in Assyrien, in: T. HölSCHER (Hg.), Gegenwelten zu den Kulturen Griechenlands und Roms in der Antike, München/ Leipzig 2000, 28 und passim. Der früheste Beleg dafür ist in Assyrien unter Tiglathpileser I. (1114-1076 v. Chr.) in dessen Palästen in Assur und Ninive zu finden, der dabei aber auf eine babylonische Tradition zurückgreifen dürfte.

27 J. Weinstein, The Egyptian Empire in Palestine, BASOR 241 (1982), 20 zum Befund in Palästina; die königlichen Statuen beginnen erst mit Sethos I. Vgl. K. A. Kitchen, Amenhotep III and Mesopotamia, in: D. und Barken, die aus Ägypten an die Königshöfe im Orient gelangten, in ihrer eigentlichen Funktion als Kultobjekte Verwendung fanden. Der Statuenexport ist deshalb keinesfalls mit „Religionsexport“ gleichzusetzen.

\section{§3. ÄgYPtische Statuen AUßERHALb ÄgyPtens}

Leider ist die Anzahl der archäologisch nachgewiesenen Statuen aus der Zeit des Neuen Reiches im asiatischen Nachbarraum eher gering. ${ }^{27}$ Die angetroffenen Statuen werden in der Regel überzeugend mit Installationen der ägyptischen Verwaltung in Zusammenhang gebracht. Sehr unterschiedlich stellt sich die Situation hinsichtlich des Mittleren Reiches dar. Allerdings ist die Verbringung ägyptischer Plastik ins Ausland auch hier äußerst problematisch und Gegenstand heftiger Diskussionen. ${ }^{28}$ Ausgangspunkt für eine kurze Rekapitulation dieser Problematik soll die vorbildliche Untersuchung von W. Helck sein, deren Ziel
O’Connor/E. H. Cline (Hg.), Amenhotep III. Perspectives on his Reign, Ann Arbor 1998, 260: Die in den Amarnabriefen beschriebenen Güter schlagen sich im archäologischen Befund kaum nieder.

${ }^{28}$ Vgl. W. A. WARD, Remarks on some Middle Kingdom Statuary found at Ugarit, UF 11 (1979), 799-806. Darauf aufbauend: G. SCANDONE MATThiae, La statuaria regale egiziana del Medio Regno in Siria, UF 16 (1984), 181-188. DiEs., Art et politique: Les images de pharaon à l'étranger, $\ddot{A} \mathcal{E} L ~ 10$ (2000), 187-193 als Reaktion auf den Ansatz von Helck. Es wird nach wie vor für eine „legale“ Verbringung der Statuen nach Syropalästina im Mittleren Reich und für eine Beibehaltung der damit verbundenen chronologischen und kulturgeschichtlichen Relevanz argumentiert. J. P. Thalmann, La civilisation des palais levantins à l'âge du bronze ancien et du bronze moyen, in: A. CAUBET (Hg.), Lacrobate au taureau. Les découvertes de Tell el-Dabca (Égypte) et l'archéologie de la Méditerranée orientale (1800-1400 av. J.-C.). Actes du colloque organisé au musée du Louvre par le Service culturel le 3 décembre 1994, Paris 1994, 112-113 geht von einem allgemeinen regelmäßigen Geschenkaustausch zwischen den Palästen der Mittleren Bronzezeit mit diplomatischem Hintergrund aus, wobei er Ägypten als gleichberechtigten Teilnehmer an dieser Kommunikation zwischen den Höfen ansieht. Aus ägyptologischer Sicht ist dazu allerdings anzumerken, daß eine Sonderstellung Ägyptens, wie sie in anderen Bereichen der Diplomatie dieser Zeit verbrieft ist (Heiratsdiplomatie), auch in diesem Zusammenhang aufgrund der unterschiedlichen Funktionalität und kulturellen Einbindung der Plastik vorstellbar und wahrscheinlich ist. 
es ist, für die Verbringung der Kunstwerke, sowohl königliche als auch private Statuen des Mittleren Reiches, einen späteren Zeitpunkt zu postulieren. ${ }^{29}$ Für Helck ist ein derartiger Vorgang einzig in der Hyksoszeit denkbar, denn die ohne Zweifel bestehende Nachfrage nach ägyptischen Statuen konnte erst befriedigt werden „als in Ägypten Fremde herrschten". 30

$\mathrm{Zu}$ diesem Ansatz ist zunächst zu bemerken, daß der allgemeine stratigraphische Zusammenhang, in dem die Statuen des Mittleren Reiches gefunden wurden, unklar ist. Es scheint die für Helck a priori feststehende Fremdheit der Hyksos zu sein, die ihn die Zäsur der Zweiten Zwischenzeit wählen lässt. $\mathrm{Zu}$ dieser Fremdheit ist freilich zu bemerken, daß die „Fremdherrschaft der Hyksos“ ein von der ägyptischen Geschichtsschreibung nachträglich eingeführter Topos ist, der nicht dermaßen unkritisch übernommen werden sollte. Greift man aus dem Fundus der in Syrien und Palästina gefundenen Statuen etwa die Sphingen heraus, so ist anzumerken, daß sich die Hyksoskönige gerade in bezug auf diese Statuengruppe verhielten wie ihre ägyptischen Nachfolger: sie usurpierten sie

${ }^{29}$ Helck, op. cit. (s. o. Anm. 2), 101-115.

${ }^{30}$ Ibid., 114.

31 Z. B. CG 393, CG 394, CG 530, CG 1243, die sogenannten Hyksossphingen aus Tanis. Vgl. B. FAY, The Louvre Sphinx and Royal Sculpture from the Reign of Amenemhat II, Mainz 1996, 14, Pls. 8c-e zur Sphinx Louvre A 23 (Amenemhet II.) mit einer Kartusche des Apophis.

32 M. BietaK, Kat.Nr. 126, in: M. BietaK/I. Hein (Hg.), Pharaonen und Fremde. Dynastien im Dunkel, Wien 1993, 150-152, zu einem Torpfosten mit einer Inschrift des Hyksoskönigs $S k r-H r$. D. Wildung, Kat.Nr. 127, in: BIETAK/HEIN, op. cit., 152, zum Schreibzeug des Apophis.

33 J. Assmann, Ägypten. Eine Sinngeschichte, München/Wien 1996, 225-227. Zu Beginn des Neuen Reiches wird das Feindbild Asien kreiert. Assmann erklärt so die ägyptische Expansion, die gewissermaßen als rechtschaffener Kampf gegen den asiatischen Seth überschrieben wird. Vgl. R. Gundlach, Der Pharao und sein Staat, Darmstadt 1998, 31-32 zur Entstehung eines ägyptischen „Weltreichsprogramms“, das letztlich dem ägyptischen Imperialismus des Neuen Reiches zugrunde liegt.

34 M. Bietak/I. Forstner-Müller/C. Mlinar, The Beginning of the Hyksos Period at Tell el-Dabca: A Subtle Change in Material Culture, in: P. Fischer (Hg.), Studies in Honour of Paul Åström, 171-181.

35 W. Helck, Statuen im Ausland, LÄ V, 1260. J. Mallet, Ras Shamra-Ougarit (Syrie). La chronologie de la période du Bronze moyen (fin du IIIe millénaire av. J.-C. et $1^{\text {re }}$ moitié du second), UF 28 (1996), 443-451. Ders., Ras Shamra-Ougarit (Syrie). La poterie du Bronze moyen durch Anbringung ihrer Kartuschen. ${ }^{31}$ Auch der epigraphische Befund zeigt, daß sich die Hyksos in der Selbstdarstellung sogar in Auaris (Tell el$\mathrm{Dab}^{\mathrm{c} a}$ ) des überkommenen ägyptisch-herrscherlichen Formenschatzes bedienten. ${ }^{32}$ Die Fremdheit der Hyksosherrscher ist somit ein Vorurteil, das sich in der aus ägyptozentrischen Quellen gespeisten Ansicht einer Eroberung durch nicht oder kaum akkulturierte Eindringlinge begründet. ${ }^{33}$ Demgegenüber konnte vor allem aufgrund der in Tell el-Dabca festgestellten materiellen Kultur der Hyksoszeit und ihrer Entwicklung die neue Sicht einer sich langsam im Delta entwickelnden Macht gewonnen werden. ${ }^{34}$ Der archäologische Befund ergibt, daß die stratigraphisch einordenbaren Statuen aus Schichtzusammenhängen der späten 18. Dynastie stammen. ${ }^{35}$ Dieser terminus ante quem würde sehr wohl auch eine Datierung in die Amarnazeit erlauben. Freilich kann dies nur als sehr vorsichtige Hypothese im Raum stehen bleiben. Dagegen spricht die allgemeine Zeitstellung der Statuen, die sämtlich aus der 12.-13. Dynastie stammen. Außerdem sind Statuen des Djefaihapi, eines Gaufürsten unter Sesostris I, sowohl in Kerma ${ }^{36}$ als

(fin du IIIe millénaire av. J.-C. et $1^{\text {re }}$ moitié du second), UF 29 (1997), 553-554 ordnet die Sphingen jetzt eindeutig einer Planierschicht unmittelbar unter dem Baaltempel zu. Sie stammen somit im weitesten Sinne aus Bauvorgängen des späteren Neuen Reiches. Einen Hinweis auf die ursprüngliche Verwendung der Statuen in einem Tempelkontext des Mittleren Reiches aus der rein zweidimensionalen Interpretation ihrer Verteilung im späteren Tempelbereich zu postulieren, hieße, den stratigraphischen Zusammenhang, die Dritte Dimension in der Archäologie, zu ignorieren. Einen allerdings noch nicht nachgewiesenen Vorgängerbau unter dem Baalstempel anzunehmen, würde ausgehend von der a posteriori feststehenden Meinung, es handle sich eben um eine Weihung treuer Vasallen der Ägypter, archäologische Befunde gewissermaßen virtuell erzeugen, und die Frage nach dem Sinn von Ausgrabung schlechthin aufwerfen. Für Informationen zur stratigraphischen Situation in Ugarit sind wir auch Y. Calvet zu Dank verpflichtet. Zu Tel Dan s. A. R. Schulman, An enigmatic Egyptian presence at Tel Dan, in: Festschrift Jürgen von Beckerath, НÄB 30, Hildesheim 1990, 235-244. Wir danken auch D. ILAN, der uns freundlicherweise Fotos der betreffenden Statuen zur Verfügung stellte.

36 Helck, op. cit. (s. o. Anm. 2), 101-102. Dabei ist zu beachten, daß die Datierung der im Tumulus gefundenen Statuen in die Hyksoszeit nicht abzusichern ist, da eine große zeitliche Streuung der Kunstwerke bis in die 18. Dynastie vorliegt. 
auch in Tell Hizzin ${ }^{37}$ zutage getreten. Die Statuen stammen aus dem Grab des Beamten in Assiut. Helck nimmt an, daß innerhalb eines sehr kurzen Zeitraums die Statuen nach Kerma und Syrien verhandelt worden wären, was aber so nicht als zwingend angesehen werden kann. Vielmehr kann eine differenziertere Sicht von Vorteil sein, die zwischen verschiedenen Statuentypen unterscheidet, nämlich. Privat- und Königsstatuen. ${ }^{38}$ Hervorzuheben ist mit Helck, daß Privat- und Königsstatuen zunächst in Ägypten selbst in Tempeln (!) aufgestellt worden waren, dann verschleppt wurden. Die meisten Privatstatuen stammen allerdings aus den Gräbern der Beamten am Heimatort. Deshalb ist ein neuerlicher Versuch, aus dem Fund der Statuen auf die Anwesenheit der Dargestellten am Fundort zu schließen ${ }^{39}$ und so eine ägyptische Machtsphäre in Syrien und Palästina zur Zeit des Mittleren Reiches zu konstruieren unter Verweis auf die „grabzentrierte Zugehörigkeitsstruktur"40 der ägyptischen Beamtenschaft abzulehnen. ${ }^{41}$ Besonders bemerkenswert ist der große Anteil der Sphingen, die, wie die zahlreichen Usurpierungen der in Ägypten aufgefundenen Stücke zeigen, sehr lange an ihrem Aufstellungsort bestanden haben dürften, d. h., sie könnten sehr wohl erst zu einem wesentlich späteren Zeitpunkt verschleppt worden sein. Hinzuweisen ist auch auf das kleine Format der meisten Statuen.

Es kann hier keine erschöpfende Diskussion dieses Themas erfolgen. Es lässt sich allerdings die Minimalaussage gewinnen, daß das Versenden von Statuen aus Ägypten nur in Extremsituationen denkbar ist; keinesfalls lässt sich eine Kontinuität des Statuenversendens vielleicht sogar schon seit dem Alten Reich annehmen. ${ }^{42}$

\section{§4. ÄGYPTISChe STATUEN IN IHREM URSPRÜNGLICHEN KONTEXT}

Es gab keinen innerägyptischen Statuenmarkt, keine „profanen“ Statuen, die auf einem wie auch immer gearteten Weg verhandelt hätten werden können. Ägyptische Plastik war - fast eine Binsenweisheit der ägyptischen Kunstgeschichte - funktionsgebunden. Die Art dieser Funktion reicht von kultisch bis funerär. Aus dieser Eigenart erklären sich auch die typischen Merkmale ägyptischer Kunst.

Anzumerken ist in diesem Zusammenhang, daß selbst profane Güter nicht durch freien Handel ins Ausland gelangten, vielmehr war jegliche Form des Austausches in hohem Maße kontrolliert und damit Teil eines politischen Kalküls seitens des Herrschers oder der Tempel. Um so mehr muss dies für eben nicht profane Güter gelten. Da die Tempel in unserem Zusammenhang nicht als Urheber in Frage kommen, bleibt ein herrscherliches Konzept als Impuls für diesen Handel. Routinemäßiges Versenden von Statuen ins Ausland ${ }^{43}$ ist aus ägyptischer Sicht nur schwer vorstellbar. Dies erklärt sich bei Götterstatuen von selbst, gilt aber auch für Königsstatuen, weil diesen ein großes Eigenleben zugeschrieben wurde und sie Anteil am Tempelkult hatten. ${ }^{44}$

Eine besondere Gruppe ägyptischer Plastik stellen die Affenfiguren, sowohl Einzeltiere als auch Gruppen von Mutter und Kind, dar. Sie können aus allen möglichen Materialien bestehen, oftmals handelt es sich um Gefäße. Auch hier ist eine gewisse Funktionsgebundenheit evident, wurden sie doch die gesamte ägyptische Geschichte hindurch offenbar an Vasallen im Ausland gesand. ${ }^{45}$ Dieser Usus scheint aus ägyptischer Sicht Ausdruck für das Untertanenverhältnis etwa des Bürgermeisters von Byblos gewesen zu sein. ${ }^{46}$ Daß gerade in der Amarnakorrespondenz ein Hinweis auf eine solche Figur vorkommt, ${ }^{47}$ zeigt die Rolle dieser Kunstwerke im Rahmen der ägyptischen „Diplomatie‘. Die Tatsache, daß seitens der Asiaten offensichtlich sogar eine gewisse Nachfrage nach den an sich erniedrigenden Geschenken bestand, ist ein Zeichen für die strikte Direktionalität ägyp-
37 Ibid., 106-107.

38 Scandone Matthiae, op. cit. (s. o. Anm. 28), 187. Thalmann, op. cit., 112 (s. o. anm. 28).

39 Scandone Matthiae, op. cit. (s. o. Anm. 28), 188-189.

40 J. Assmann, Herrschaft und Heil. Politische Theologie in Altägypten, Israel und Europa, München/Wien 2000, 232.

${ }^{41}$ Ibid., 229-232.

42 Dagegen Scandone Matthiae, op. cit. (s. o. Anm. 28), 192.
43 Ibid., 190 nimmt dies für das Mittlere Reich an.

${ }^{44}$ M. Müller, Die Kunst Amenophis' III. und Echnatons, Basel 1988, III, 1-2.

45 Fischer, op. cit. (s. o. Anm. 16), 8-9.

${ }^{46}$ Ibid., 6-7. Die Belege stammen v. a. aus der Sechsten Dynastie. Eine Häufung derartiger Statuen und Gefäße fand sich im Ba'alat/ Hathor-Tempel in Byblos.

${ }^{47}$ S. o., §2 zu EA 14 ii 48. 
tischer Kommunikation mit dem Ausland und für die unterschiedliche Wahrnehmung der kulturellen Kontexte, was Ägypten zu nutzen wußte, um mit einer Gabe einerseits Dankbarkeit und Einfluß im Ausland zu gewinnen und andererseits im Sinne der innenpolitischen Propaganda des Neuen Reiches ägyptische Hegemonie über wesentliche Teile Asiens zu postulieren. Die Freude der Beschenkten findet eine amüsante Parallele in Karl dem Großen, der Harun al-Rashids Geschenk eines Elephanten und eines Mantels gerne akzeptierte, ohne zu ahnen daß er sich damit zu einem „humble servant“48 machte.

Außerdem ist die Einstellung der Ägypter zur Fremde zu bedenken, wie sie beispielsweise in der Erzählung des Sinuhe evident ist. ${ }^{49}$ Ganz besonders problematisch ist aus ägyptischer Sicht das asiatische Ausland als Inbegriff des sethisch-feindlichen Fremdlandes,$^{50}$ und der Export von Statuen dorthin nur in Ausnahmefällen denkbar. Besonders bemerkenswert ist in diesem Zusammenhang ein Brief vom Hofe Merenptahs an den König von Ugarit als Reaktion auf dessen Bitte, ihm ägyptische Künstler zu senden. ${ }^{51}$ Die Künstler waren angefordert worden, um eine Statue Merenptahs anzufertigen, die sodann im Baalstempel von Ugarit aufgestellt werden sollte. Nach einer Verzögerung seitens der Ägypter, die wiederum sehr genau den diplomatischen Gepflogenheiten der Amarnakorrespondenz entspricht, ${ }^{52}$ wird die Entsendung von Zimmerleuten in Aussicht gestellt. ${ }^{53}$ Daraus ist zu ersehen, daß die betreffende Königsstatue aus Holz hergestellt werden sollte; ein entsprechendes Stück wäre leicht genug aus Ägypten nach Ugarit zu transportieren gewesen. Aus diesem Brief ergibt sich somit nicht nur ein Hinweis auf die große
Nachfrage nach ägyptischer Plastik und ägyptischem know how in der Bildhauerei in Vorderasien, sondern auch auf die Notwendigkeit komplizierter Manöver, um das Verschicken einer ägyptischen Königsstatue zu umgehen.

Die Art der Kunstwerke, die von Ägypten nach Vorderasien verschickt wurden, lässt sich aus der Amarnakorrespondenz erschließen. Auffällig ist dabei der große Anteil kleinformatiger, teilweise vergoldeter Bildwerke, und hinzuweisen ist besonders auf die hölzernen Schiffe" ${ }^{54}$ bei denen es sich um Götterbarken handeln dürfte. Hier ist der ursprüngliche sakrale Kontext am deutlichsten, aber auch in den meisten anderen Fällen dürfte es sich aufgrund des beschriebenen generellen Charakters ägyptischer Kunstproduktion um ganz ähnliche Zusammenhänge handeln. Die Hypothese, daß die meisten der exportierten Statuen zum Tempelinventar im engeren oder weiteren Sinne gehört haben dürften, lässt an den oben erwähnten Prozessionskontext anknüpfen. ${ }^{55}$ Besonders ist an die Funktion des ägyptischen Tempels als Mechanismus, der das Funktionieren des Kosmos gewährleistet, zu erinnern,${ }^{56}$ die Rolle, die Götter- und andere Bilder darin spielten, ist nicht zu unterschätzen. ${ }^{57}$

Der ägyptische Kontext lässt sich also in den Fällen von Königs- und Götterstatuen - und um solche dürfte es sich hauptsächlich handeln - als Tempelkontext beschreiben. Der in der Amarnakorrespondenz erfasste Zeitraum beginnt mit dem 13. Regierungsjahr Amenophis' III. und endet mit dem Ende der Amarnazeit. ${ }^{58}$ Deshalb sollte eine Betrachtung der Herkunftsumstände der Statuen die Entwicklungen von der Regierungszeit Amenophis' III. an berücksichtigen und auch diese Epo-

48 P. Brown, The World of Late Antiquity, London 1989, 203.

49 Assmann, op. cit. (s. o. Anm. 40), 217-242.

${ }^{50}$ S. o. Anm. 33.

51 RS 88.2158, s. S. LACKENBACHER, Une corréspondance entre l'administracion du Pharaoh Merneptah et le roi d'Ougarit, in: P. Bordereuil/M. SzNYCER/M. Yon (Hg.), Le Pays d'Ougarit autour de 1200 av. J.-C. Histoire et archéologie. Ras Shamra Ougarit XI, Paris 1995, $77-83$.

52 M. Liverani, The Great Power's Club, in: Cohen/ WestBROOK, op. cit. (s. o. Anm. 3), 24.

53 Assmann, op. cit. (s. o. Anm. 40), 231 hebt die alleinige Verfügungsgewalt des Königs über die Handwerker hervor und bezeichnet diese als ganz wesentlichen

Machtfaktor. Das Hinauszögern des Entsendens und schließlich die Reduktion der Handwerker auf Tischler kann möglicherweise als ein Versuch Merenptahs verstanden werden, den König von Ugarit seine Macht spüren zu lassen.

${ }^{54}$ S. o., § 2, zu EA 14 ii 17-18.

55 S. o. Anm. 75 und vgl. J. Kemp, Ancient Egypt. Anatomy of a Civilization, London/New York 1991, 185-188 zur großen Bedeutung der Prozessionen im sozialen Gesamtkontext.

56 Egberts, op. cit. (s. o. Anm. 5), 13.

57 D. Arnold, Die Tempel Ägyptens, Augsburg 1996, 13-14, 49.

58 S. o. Anm. 7. 
che für die Neuerungen der Amarnazeit bezüglich des Tempelkultes in Betracht ziehen. ${ }^{59}$

Bemerkenswert scheint zunächst die Tatsache, daß gerade Amenophis III. besonders viele Bildnisse in die Tempel stiftete. Aus der Amarnakorrespondenz ist weiters klar, daß bereits Amenophis III. mit dem Verschicken der Statuen begann. Der Beginn des Umbruchs dürfte also schon gegen Ende der Regierung Amenophis' III. erfolgt sein. ${ }^{60}$ Unter Amenophis IV. zeichnen sich dann dramatische Veränderungen ab, eine Revolution von oben, die sich in einer gewaltsamen Ablehnung der traditionellen Kulte äußerte und dazu führte, daß das traditionelle Ägypten zu jenem „Außen“ wurde, das zuvor die Asiaten dargestellt hatten. Die Bekämpfung der alten Kulte und Götter wurde zur heiligen Aufgabe des Königs als Bewahrer der Maat. ${ }^{61}$ Möglicherweise sind die weniger aggressive Haltung Ägyptens gegenüber Asien $^{62}$ und verschieden fremdländische Eigenheiten während der Amarnazeit zum Teil auf diese Änderung des königlichen Selbstverständnisses zurückzuführen. ${ }^{63}$

Diese neue Herrschaftsideologie äußert sich auch in einem gemeinsamen Machtgewinn von König und Sonnengott, der in der Amarnazeit seinen Höhepunkt erreicht. Hinsichtlich des Sonnengottes lässt sich die Entwicklung anhand der Schöpfung einer neuen Sonnentheologie, die sich vor allem in den erhaltenen Sonnenhymnen entfaltet, ${ }^{64}$ verfolgen. ${ }^{65}$ Zwar gibt es unter Amenophis III. sehr

${ }^{59}$ Vgl. R. E. Freed, Y. J. Markowitz, S. H. D’Auria (Hg.), Pharaohs of the Sun, Boston 1999, für eine Zusammenfassung des allgemeinen kulturellen Milieus der Zeit Amenophis' III. und Echnatons.

${ }^{60}$ Anders W. R. Johnson, Monuments and Monumental Art under Amenhotep III, in: O'Connor/Cline, op. cit. (s. o. Anm. 27), 93, der vorschlägt, die alleinige Herrschaft des Atonkultes wäre eine moderne Verkürzung, die auf dem Zufall der guten Quellenlage in Echnatons Hauptstadt beruhe. Die Amarnarevolution und der Kampf gegen Amun wären erst nach dem Tod Amenophis' III. denkbar. Allerdings ist für das Verständnis der Amarnazeit doch Amenophis III und seine „increasing solarization“ nicht wegzudenken, s. ibid., 94. Vgl. J. Baines, The Dawn of the Amarna Age, in: O'Connor/Cline, op. cit., 271-272.

${ }^{61}$ J. Baines, Kingship, Definition of Culture and Legitimation, in: D. O'Connor/D. P. Silverman (Hg.), Ancient Egyptian Kingship, Leiden/New York/Köln 1995, 28.

62 Assmann, op. cit. (s. o. Anm. 33), 229 spricht von einem „Einbruch, den die Amarnazeit für die ägyptische Asienpolitik bedeutet hat". wohl noch andere Götter, doch ist ihre Rolle statistenähnlich eingeschränkt, das nicht Amun Re Seiende wird zu einem undifferenzierten Kollektiv, dem letztlich auch die Menschen angehören ${ }^{66}$ Es kommt also zu einem Herausschälen der Macht eines Gottes, zu einer Art Individualisierung. Damit ändert sich auch die Vorstellung vom Kosmos, ist doch der Sonnengott bei seinem Sonnenlauf nicht mehr in das enge Korsett aus Anforderungen, Ritualen, letztlich Gefahren ${ }^{67}$ gebunden. In unserem Zusammenhang bedeutet dies, daß sich auch die irdische Immanation dieses kosmischen Mechanismus, der Tempel und sein Kult, veränderte. Der häufig geäußerten Feststellung, daß es in der Zeit Amenophis' III. noch zu keiner Veränderung der Tempelarchitektur gekommen wäre, ${ }^{68}$ ist entgegenzuhalten, daß es, was den Statuenschmuck der Tempel, und da vor allem die Königsstatuen betrifft, zu massiven Neuerungen kommt. So verlagert sich das Schwergewicht offensichtlich vom hinteren, intimeren Tempelbereich mit den dort aufgestellten Kleinformaten hin zu repräsentativer Monumentalplastik in den vorderen, „politischen“ Abschnitten. Die Versendung einer vergoldeten Statue seiner selbst, seiner Frau und seiner Tochter ${ }^{69}$ als diplomatisches Geschenk ist möglicherweise mit einem Nachlassen der Funktionsgebundenheit gerade solcher Bildwerke im kultischen Bereich verbunden.

Die Veränderung der Position des Königs unmittelbar vor der Amarnazeit ${ }^{70}$ entspricht in vie-

${ }^{63}$ Schlagenster Beweis für völlig unägyptisches herrscherliches Verhalten ist wohl der Brief einer nicht näher zu bestimmenden, sicherlich aber dem Amarnabereich zuzuordnenden Königin an den Hethiterkönig mit der Bitte um Sendung eines Prinzen zwecks Ehe und Erhaltung der Dynastie. Vgl. F. J. GiLEs, The Amarna Age. Western Asia, Warminster 1997, 311-318; Liverani, op. cit. (s. o. Anm. 3), 278-279. Die Rolle von Königin und Königsmutter während der Amarnazeit erinnert an die hethitischen Gepflogenheiten (vgl. z. B. die Rolle der Königin in der zwischenstaatlichen Korrespondenz).

${ }^{64}$ BAINES, op. cit. (s. o. Anm. 60), 277-279, eher relativierend zu einer direkten Abhängigkeit der Amarnatheologie von solchen Vorläufern.

${ }^{65}$ Assmann, op. cit. (s. o. Anm. 33), 235-242.

${ }^{66}$ Ibid., 242. Ebenso BAINES, op. cit. (s. o. Anm. 60), 280.

${ }^{67}$ Assmann, op. cit. (s. o. Anm. 33), 236.

${ }^{68}$ Z. B. BAINES, op. cit. (s. o. Anm. 60), 296.

${ }^{69}$ S. o., §2, zu EA 14 ii 11-14.

${ }^{70}$ W. R. Johnson, The Setting: History, Religion and Art, in: Freed/Markowitz/D'Auria, op. cit. (s. o. Anm. 59), 42-45. 
lerlei Hinsicht jener des Sonnengottes. Zeichen dieser Entwicklung sind einmal die Kolossalstatuen, die unter Amenophis III. in nie dagewesenem Ausmaß entstehen ${ }^{71}$ und das Landschaftsbild über weite Strecken geprägt haben dürften. Weiters zu nennen ist die neue Vielfalt unterschiedlicher menschlicher Qualitäten, etwa Altersstufen, in denen der Pharao erscheint. Schließlich sei noch auf die Bezeichnung des Königs als $j t n-t h n^{72}$ auf dem großen Skarabäus in Luxor hingewiesen, also auf die Gleichsetzung des Königs mit der Sonnenscheibe. Der König nähert sich der göttlichen Sphäre damit weitestgehend an, was sich auch in den Kulten seiner Statuen zeigt, ${ }^{73}$ und damit bietet sich eine mögliche Erklärung für die gleichzeitige Machtbildung von Amun-Re und König an: Der König formte den Gott um, weil er sich nur einem Ideal angleichen konnte, das selbst optimale Machtbefugnis und geringstmögliche Abhängigkeit von Mitgöttern oder Menschen zeigt. $^{74}$ Deutlich wird dies in der Stellung der Göt-

71 Baines, op. cit. (s. o. Anm. 60), 294-295.

72 Ibid., 291 Anm. 68.

73 Müller, op. cit. (s. o. Anm. 44), III 13 Anm. 6 zur Darstellung eines Opfers vor einem der Memnonskolosse. Ibid., III 14 zur Rolle des nördlichen Memnonskolosses als Schutzgottheit. M. EL SAGHIR, Das Statuenversteck im Luxortempel, Mainz 1992, 21-27 zur Darstellung einer auf dem Schlitten stehenden Statue des Amenophis III. S. auch Johnson, op. cit. (s. o. Anm. 60), 68. Vgl. BAInes, op. cit. (s. o. Anm. 61), 26 zur zunehmenden Annäherung Amenophis' III. an die Göttlichkeit.

74 A. VAN GENnEP, Übergangsriten, Frankfurt 1986, 179. Die fast mechanistische Vorstellung des Zusammenhangs von Ritus und Funktionieren des Sonnenlaufs schränkte den Sonnengott und damit den König, der sich ihm mehr und mehr anglich, ein. Der Tempelkult und die mit ihm verbundenen Hierarchien wurden zu einem Widerspruch zu königlicher und göttlicher Macht. Vgl. S. Morenz, Agyptische Religion, Stuttgart 1960, 42, der anmerkt, daß das Königtum ,den Umgang mit den Göttern gewährte, aber den Vollzieher des Kultes zugleich in die Knie zwang".

75 Müller, op. cit. (s. o. Anm. 44), III 32, III 35. Vor allem anhand der momiformen Statuen im Totentempel läßt sich eine zunehmende Emanzipation des menschlich/ königlichen gegenüber dem osirianischen feststellen. Danach kommt es in der Amarnazeit zu völligen Neuschöpfungen, wie freistehenden momiformen Statuentypen. Besonders deutlich wird der herrschaftliche Anspruch des Königs an Details der Statuen. Ähnlich dem Djoserbezirk stellt auch der Totentempel Amenophis' III. einen Mikrokosmos Ägyptens dar. Dies äußert sich sowohl in der Gestaltung der Kronen (oberägyptische Krone bei den südlichen, unterägyptische bei den terversammlung bei den Sedfesten Amenophis' III., wie sie durch in den Resten des Totentempels ${ }^{75}$ des Königs erhaltene Spuren rekonstruierbar ist. ${ }^{76} \mathrm{Ganz}$ ähnlich wie oben für das göttliche Wirken des Sonnengottes geschildert, wohnen die Götter als Kollektiv dem König und seinem Tun bei und gesellen sich zu den menschlichen Zuschauern. Diese Sedfeste zeigen wiederum die teilweise durchaus evidenten archaisierenden Züge in der Zeit Amenophis' des III. ${ }^{77}$ Amenophis IV findet hier das unmittelbare Vorbild für seine eigenen Feiern. ${ }^{78}$

Die Zeit Echnatons stellt in vielerlei Hinsicht einen Kulminationspunkt dieser Entwicklungen $\mathrm{dar},{ }^{79}$ obwohl der revolutionäre Charakter der einzelnen Aktionen Echnatons keinesfalls ignoriert werden darf. ${ }^{80}$ Viele Merkmale der Regierung Echnatons lassen sich mit dem von Max Weber kreierten Begriff der „Charismatischen Herrschaft" ${ }^{\text {"81 }}$ beschreiben. Typisch ist sowohl der starke Anfangsimpuls, der den charismatischen Führer gemein-

nördlichen Osirispfeilern) als auch beim Material (Rosengranit bei den südlichen, Quarzitkalkstein bei den nördlichen Osirispfeilern). J. GOHARY, Akhenaten's Sed-Festival at Karnak, London/New York 1992, 167-168 vermutet eine dualistische Anordnung der Szenen des Sedfestes, im Süden Ober-, im Norden Unterägypten wobei der König die ensprechende Krone trägt. Vgl. E. Cline, Amenhotep III, the Aegean and Anatolia, in: O'Connor/Cline, op. cit. (s. o. Anm. 27), 236-239 zur Beschriftung einer im hinteren Teil des Totentempels gefundenen Statuenbasis mit einer Liste ägäischer Fremdvölker, die als Unterworfene dargestellt sind. Dieser Fund beweist, daß Amenophis III. den ägyptischen Weltreichsanspruch beträchtlich erweitert.

76 Müller, op. cit. (s. o. Anm. 44), III 55. Johnson, op. cit. (s. o. Anm. 60), 74-76.

77 E. Hornung, Sedfest und Geschichte, MDAIK 47 (Festschrift für Werner Kaiser) (1991), 171 hebt die herausragende Bedeutung des Sedfestes unter Amenophis III. hervor.

78 Gohary, op. cit. (s. o. Anm.75), 167-168. Echnaton wollte das Sedfest so traditionell wie möglich gestalten, ersetzte die an sich notwendigen und bei seinem Vater selbstverständlich noch anwesenden Götter durch Aton, der in verschiedene Rollen schlüpfte.

79 Morenz, op. cit. (s. o. Anm. 74), 42-43 spricht von einem allgemeinen Spannungszustand zwischen Menschlichkeit und Göttlichkeit des Königs und stellt einen Zusammenhang zur Vergöttlichung des Königs in der Amarnazeit fest. Vgl. BaInes, op. cit. (s. o. Anm. $60), 312$.

${ }^{80}$ Baines, op. cit. (s. o. Anm. 60), 307-311.

${ }^{81}$ M. WEBER, Wirtschaft und Gesellschaft, Tübingen $1972^{5}$, 654-681. 
sam mit einer hochmotivierten Elite ${ }^{82}$ an die Macht bringt (wobei diese Motivation vor allem emotionaler Natur und von hoher Energieentwicklung ${ }^{83}$ begleitet ist, wie die gewaltsame Durchsetzung der neuen Religion zeigt ${ }^{84}$ ), als auch die geringe Stabilität und die Schwierigkeit, die mit der Legitimation solcher Herrschaft verbunden ist. ${ }^{85}$

Diese Problematik lässt sich anhand einiger Detailprobleme der Amarnazeit zeigen. Unter Amenophis IV. kommt es zu einem Aufheben der alten Begriffe von Innen und Außen nicht nur im politischen, sondern auch im kultischen Bereich. Dies zeigt sich in einer neuartigen Veröffentlichung des Kultes, die sich z. B. in der offenen unüberdachten Tempelarchitektur manifestiert. Diese neue Öffentlichkeit aber zeitigt deutliche Auswirkungen auf wesentliche Kultgebräuche. Verschiedene Installationen, deren Existenz vor allem in der Unzugänglichkeit des täglichen Rituals begründet war, wurden obsolet bzw. erfuhren eine Umwertung.

Eine solche Einrichtung sind die Prozessionen, einer der wesentlichsten identitätsstiftenden Aspekte der ägyptischen Gesellschaft. ${ }^{86}$ Die ägyptische Festreligion scheint zu einem Gutteil durch inszeniertes Interagieren von königlicher Familie und Aton ${ }^{87}$ ersetzt worden zu sein. Damit dürfte die zeremonielle Funktion von Teilen des Palastes in Amarna zusammenhängen. ${ }^{88}$ Diese Veränderung wirkte sich natürlich auch auf den Statuenschmuck der Tempel aus. Die im hinteren Bereich des Tempels aufbewahrten kleineren, meist tragbaren Statuen nebst den Barken hatten im neuen Kultgeschehen keinen Platz mehr. Besonders bemerkens- wert ist in diesem Zusammenhang auch das fast vollständige Fehlen von Sphingen, einer noch unter Amenophis III. sehr beliebten Statuengattung. Dies lässt sich möglicherweise mit der speziellen Funktion der Sphingen erklären, die durch ihren steingewordenen Übergangszustand zwischen menschlich/göttlich/animistischer Sphäre zu einem Kürzel für das ägyptische Kultverständnis werden. Offenbar wird diese Funktion der Membran zwischen Innen- und Außenraum des Tempels einerseits durch den Aufstellungsort vor den Tempeln ${ }^{89}$, andererseits durch die bis in späteste Zeit überlieferte Orakelfunktionen einzelner Statuen dieser Gattung. Bemerkenswert sind in diesem Zusammenhang zwei Reliefblöcke mit Sphingendarstellungen aus Amarna. ${ }^{90}$ Die Sphingen zeichnen sich durch eindeutiges Interagieren mit der Sonnenscheibe aus. In der einen Darstellung opfert der Sphinx, versehen mit den Zügen Echnatons, der Sonne, deren Strahlen ihn zu liebkosen scheinen. ${ }^{91}$ Auf dem zweiten Block ist die Kommunikation zwischen Aton und dem als Sphinx dargestellten König ähnlich intim; hier handelt es sich aber um das Abbild einer Sphinx - Statue. ${ }^{92}$ Daß solche Darstellungen in situ im Palast von Amarna gefunden wurden, ${ }^{93}$ ist ein zusätzlicher Hinweis auf die sakrale Aura der Wohnstatt des Königs. Anzumerken ist hinsichtlich dieser Sphingen auch, daß es sich nicht um Rundplastiken handelt; die vermutliche Anbringung an den Innenseiten von Durchgängen erinnert stark an die orientalischen Türleibungslöwen. Zudem ist die sehr animierte Darstellung sonst nicht für Sphingen üblich.
${ }^{82}$ BAINES, op. cit. (s. o. Anm. 60), 280 spricht von einer Diskussion innerhalb einer ,Elite , aus der schließlich die Amarnazeit hervorgegangen sei.

83 Johnson, op. cit. (s. o. Anm. 70), 47: „The emotional element in Akhenaten's suppression of Amen is perplexing...".

${ }^{84}$ Weber, op. cit. (s. o. Anm. 81), 655. Gerade das ,Neue und Unerhörte reicht bereits aus, um zu überzeugen: vielleicht kommt es gerade deshalb zu den Eigenheiten der Erscheinungsform der neuen Herrschaft Echnatons. „Das Charisma kennt nur innere Bestimmtheiten und Grenzen seiner selbst“, s. WEBER, op. cit., 655. Vgl. auch ibid., 659 zur Herausbildung einer Aristokratie aus dieser Elite.

${ }^{85}$ Ibid., 661, 671. Die „Veralltäglichung“ des Charisma ist nur möglich, wenn die Gründung einer Dynastie gelingt, ansonsten endet es mit dem Tod des Herrschers.

${ }^{86}$ Assmann, op. cit. (s. o. Anm. 40), 233.
${ }^{87}$ Vgl. G. STEIndORfF, Die Kunst der Ägypter, Leipzig 1928, 74: „Mitten hinein in den Palast führt das Bild, das die Königliche Familie unter den segnenden Strahlen des Aton in zärtlicher Gemeinschaft zeigt...".

88 Müller, op. cit. (s. o. Anm. 44), III 64-65. Vgl. Baines, op. cit. (s. o. Anm. 60), 298, der eine ähnliche Entwikklung bereits für den Palast Amenophis' III. in Malkatta annimmt. Dort ist sicherlich bereits in der Zeit Amenophis' III., für die ein sehr intimer Zusammenhang zwischen Totentempel und Palast in Malkata anzunehmen. Vgl. auch o. Anm. 75 zur Entwicklung der momiformen Osirispfeiler von Amenophis III. bis zur Amarnazeit.

${ }^{89}$ VAN GenneP, op. cit. (s. o. Anm. 74), 30.

90 P. Lacovara, in: Freed/Markowitz/D'Auria, op. cit. (s. o. Anm. 59), 231, nos. 89-90.

91 Ibid., no. 90.

${ }^{92}$ Ibid., no. 89.

${ }_{93}$ Ibid., 231, Anm. 4. 
Die These von in der Amarnazeit ins Ausland verbrachten Statuen lässt sich nun aus ägyptischer Sicht teilweise passiv untermauern, indem man davon ausgehen kann, daß ein aufgrund der geänderten Kultpraxis anfallender Statuenüberschuss abgebaut wird, andererseits ist aber auch mit gewaltsamen Plünderungsaktionen zu rechnen. Gerade die Versendung der Barken würde gut in den Zusammenhang einer sich bildenden charismatischen Herrschaft passen. Derart ostentative Akte der Schmähung des Althergebrachten würden dem „Leistungsanspruch“ im Sinne von Max Weber, der an eine solche latent instabile Regierungsform gestellt wird, in höchstem Maße gerecht werden.

\section{§5. SChlussbetrachtung}

Betrachtet man die grundlegenden Rahmenbedingungen, so sind zwei Voraussetzungen ausschlaggebend: Einerseits ist dies die Nachfrage nach ägyptischer Plastik seitens der vorderasiatischen Herrscher, die sich ja bereits für wesentlich frühere Zeiträume nachweisen lässt, andererseits ein Statuenüberschuss in Ägypten, der sich im Laufe der Zeit verringerte. ${ }^{94}$

Dieser Überschuss, vor allem sein erstmaliges Auftreten, ist aus ägyptischer (ägyptologischer) Sicht besonders interessant, bieten uns doch die schriftlichen Quellen der Abnehmerseite Gelegenheit, sowohl das Ausmaß des Exports zu Beginn des Austauschs von Kunst gegen Einfluss als auch die zunehmend unbefriedigte Nachfrage im Verlauf der Amarnazeit zu beobachten. Die zu treffende Minimalaussage ist, daß ein bestehender Überschuss passiv abgebaut wurde, ohne daß die zu vergebenden Bestände durch zusätzliche Produktion aufgestockt wurden.

Das Phänomen des noch lange im ägyptischen Kunstschaffen nachhallenden Echos der Amarna- zeit, ${ }^{95}$ man denke an ikonographische Besonderheiten, wie Halsfalten oder perforierte Ohrläppchen, findet vor dem oben beschriebenen Hintergrund einer Zerschlagung der handwerklichen und künstlerischen Traditionen ${ }^{96}$ die ihre Heimat wohl vor allem im Tempelkontext hatten, eine neue Erklärung. Betrachtet man etwa die Werkstatt des Tuthmosis in Amarna und die in ihr aufgefundenen Bildhauermodelle, ${ }^{97}$ so wird anhand der wohl vom königlichen Auftraggeber geforderten Korrekturen (noch heute deutlich als rote oder schwarze Markierungen zu erkennen) klar, daß gerade jene Merkmale auf Echnatons politisches Wollen zurückgehen, die, entgegen üblicher ägyptischer Kunsttraditionen, das Individuelle des Königs und seiner Familie ausdrücken sollen. Das Herausschälen des Königs aus dem kosmisch menschlichen Kollektiv entspricht der Ausschließlichkeit des Monotheismus der Amarnazeit im Atonkult. Dieses Phänomen einer parallelen Herausbildung singulärer göttlicher und menschlicher Macht wurde bereits ausgeführt. ${ }^{98}$ Wie sehr die Amarnarevolution Ägypten zumindest unterschwellig für alle Zeiten prägte, wird gerade in diesen anscheinend nur oberflächlichen „Spurenelementen“ deutlich. Ohne diese Beobachtung in ihrer kunsthistorischen Dimension erkunden zu wollen, sei zumindest darauf hingewiesen, daß sie geeignet ist, neben den offensichtlichen quantitativen Phänomenen ${ }^{99}$ auch die bereits zitierte Assmannsche "psychologische“ Seite des Problems zu beleuchten. Die Feststellung, daß sich selbst Ramses II., der mächtigste Pharao des Neuen Reiches, bei der Gestaltung seines Sarkophages (im ägyptischen Sinne unmittelbarer Nahbereich), dessen Amarnazüge frappant sind, diesem Nachhall nicht entziehen konnte/wollte, mag unsere Darstellung abrunden. ${ }^{100}$
${ }^{94}$ S. o., §2, zu den nachdrücklichen Forderungen der vorderasiatischen Herrschern nach Erfüllung der entsprechenden Versprechen Amenophis' III. und Echnatons zögerlichem Umgang mit diesen Ansprüchen.

95 SteindorfF, op. cit. (s. o. Anm. 87), 75-77. C. Aldred, Egyptian Art, London 1980, 183-186; R. E. FreED, Akhenatens Artistic Legacy, in: Freed/Markowitz/D'AuRIA, op. cit. (s. o. Anm. 59), 187-197.

96 Freed, op. cit., 188 und 193, diskutiert die Problematik der in der Amarnazeit ausgebildeten Künstler und der neu entstandenen Tradition, die freilich „...surrounded in Thebes and Memphis by fifteen hundred years of tradition..." (ibid., 193) die Amarnaeinflüsse nur noch in sehr abgeschwächter Form in ihre Kunst einfließen ließen.

97 Vgl. den Kopf der Nofretete (Berlin Ägyptisches Museum, 21352).

98 S. o., $\$ 4$.

99 So steht etwa die Präsenz von Statuen des Tutanchamun in den großen Tempeln Ägyptens in keinem Verhältnis zu Regierungszeit und Bedeutung dieses Königs, vielmehr dürfte es sich um ein notwendiges Auffüllen des unmittelbar zuvor entstandenen Vakuums handeln. Wir danken Manfred Bietak für diesen Hinweis.

${ }^{100}$ CG 61.020, Pl. XXI. 